Z Herz-Thorax- Gefäßchir 2009 · 23:195

DOI 10.1007/s00398-009-0734-9

๑) Springer-Verlag 2009

\author{
A. Markewitz \\ Abt. XVII - Herz- und Gefäßchirurgie, Bundeswehrzentralkrankenhaus, Koblenz
}

\section{Packen wir es an!}

\section{Fokustagung Herz der DGTHG}

„Große Ereignisse werfen ihren Schatten voraus“, in diesem Fall die erste Fokustagung Herz der DGTHG vom 1. bis 3. Oktober 2009, und sie tut es in diesem Heft mit Publikation der Abstracts, die für eine dortige Präsentation akzeptiert werden konnten. Die Lektüre der Abstracts sollte Sie als Leser dieser Zeitschrift schlussendlich motivieren, sich mit der Thematik eingehend zu befassen und darüber hinaus an der ersten Fokustagung Herz in Duisburg teilzunehmen.

Hintergründe und Anlass, die zum Entschluss der DGTHG führten, neben der traditionellen Jahrestagung im Frühjahr ab diesem Jahr im Herbst eine weitere Tagung zu ausgewählten Themenbereichen $\mathrm{zu}$ veranstalten, finden Sie im Flyer zur Fokustagung Herz 2009: Allein die Zahl von ca. 100.00o Patienten, die jedes Jahr mithilfe der Herz-Lunge-Maschine operiert und auf herzchirurgischen Intensivstationen behandelt werden sowie weitere 25.00o Patienten, denen von Herzchirurgen Herzschrittmacher, Defibrillatoren und kardiale Resynchronisationssysteme implantiert, gewechselt oder revidiert werden, sollten Anlass genug sein, ein besonderes Forum für diese Themen zu schaffen. Im Rahmen der Jahrestagungen war angesichts der Vielfalt anderer interessanter herzchirurgischer Thematiken selten hinreichend Zeit und Raum, die oben genannten Inhalte zu fokussieren und sie mit der gebotenen Ausführlichkeit zu diskutieren. Das soll sich mit der Fokustagung Herz ab Oktober 2009 ändern.

In den vergangenen Wochen und Monaten haben die Mitglieder der Arbeitsgruppen „Herz- und thoraxchirurgische Intensivmedizin“, „Extrakorporale Zirkulation und mechanische Kreislaufunter- stützung“ sowie der neu gegründeten AG „Elektrophysiologische Chirurgie“ ein interessantes Programm zusammengestellt, von dem alle Beteiligten überzeugt sind, dass es den Ansprüchen einer praxisnahen Tagung gerecht wird.

Sie als Leser können sich schnell selbst einen detaillierten Überblick zu den genauen Programminhalten auf der Homepage der Fokustagung (http://www.dgthgfokustagung.de) verschaffen. Hierbei wird die generelle Ausrichtung der Tagung klar: Neben den Übersichtsvorträgen mit Vermittlung aktueller medizinischer Evidenzen stehen vor allem die tägliche Praxis in allen drei Teilbereichen und aktuelle Diskussionen über Einsatzmöglichkeiten moderner Technologien nebst dazu notwendiger Qualifikation im Mittelpunkt; kurz gesagt: „how to do“ und „who's in charge".

Die Fokustagung soll jedoch auch zur Standortbestimmung dienen. Akzeptanz bzw. Teilnahme an der Fokustagung werden zeigen, ob wir Herzchirurgen die Intensivmedizin, die extrakorporale Zirkulation, die mechanische Kreislaufunterstützung und die Therapie mit Herzschrittmachern, Defibrillatoren und kardialen Resynchronisationssystemen auch in Zukunft als herzchirurgische Kernkompetenzen verstehen. Das Organisationsteam geht davon aus, dass bei unseren herzchirurgisch tätigen Kolleginnen und Kollegen sowohl der intensive Wunsch als auch die Bereitschaft vorhanden sind, unseren Partnern in Anästhesie, Kardiotechnik, Kardiologie, Elektrophysiologie und Chirurgie, ein kompetenter Gesprächspartner bei allen drei Themen zu sein und sich den Diskussionen zu stellen. Hierauf gründet sich unsere Erwartung, eine große Zahl an Teilnehmern aus der Sparte Herzchirurgie bei der Fokustagung Herz begrüßen zu dürfen. Darüber hinaus hoffen wir auf die zahlreiche Teilnahme von Vertreter aus unseren Partnerdisziplinen, damit nach einem regen interdisziplinären Gedankenaustausch und lebhaften Diskussionen jeder Teilnehmer am 3. Oktober 2009 mit neu gewonnen Erkenntnissen und möglicherweise erweitertem Horizont den Heimweg antritt, die Teilnahme als lohnenswert ansieht und sich den selben Termin für die zweite „Fokustagung Herz" am selben Ort im Jahre 2010 bereits fest vormerkt.

In diesem Sinne verbleibe ich, stellvertretend für das gesamte Organisationsteam, bis zum Zusammenkommen am 1. Oktober in der Mercatorhalle Duisburg mit dem Motto der Fokustagung Herz: „Packen wir es an!“

Ihr

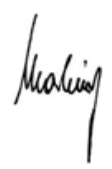
A. Markewitz

\section{Korrespondenzadresse \\ OFA Prof. Dr. A. Markewitz}

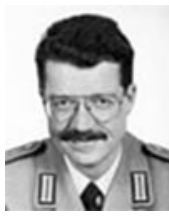

Abt. XVII - Herz- und Gefäßchirurgie, Bundeswehrzentralkrankenhaus Rübenacher Str. 170, 56072 Koblenz andreasmarkewitz@ bundeswehr.org 